\title{
Deep Neck Space Abscess - A Case Report
}

\author{
Priya Kanagamuthu¹, Guna Keerthana Ramesh², Aswin Vaishali Natarajan³, Rajasekaran Srinivasan ${ }^{4}$ \\ 1, 2, 3,4 Department of Otorhinolaryngology, Chettinad Hospital and Research \\ Institute, Kelambakkam, Tamil Nadu, India.
}

\section{INTRODUCTION}

Deep neck spaces are regions of loose connective tissue present between three layers of deep cervical fascia, namely, superficial, middle, and deep layers. The investing layer is the superficial layer, the pre-tracheal layer is the intermediate layer, and the prevertebral layer is the deep layer. Deep neck space infection (DNI) is defined as an infection in the potential spaces and actual fascial planes of the neck. Spread of infection occurs along communicating fascial boundaries. These deep neck spaces may be further classified into 3 anatomic groups, relative to the hyoid bone: Those located above the level of the hyoid, those that involve the entire length of the neck, those located below the level of hyoid. The patterns of infection may include abscess formation, cellulitis, and necrotizing fasciitis. Antibiotics and surgical drainage form the mainstay of treatment.

There are some spaces in the neck present between these layers of deep cervical fascia. These deep neck spaces are filled with loose connective tissue. Deep neck space infection involves the spaces and fascial planes of the neck. Spread of infection occurs along communicating fascial boundaries after overcoming the natural resistance of the fascial planes. With relation to the hyoid bone, these deep neck spaces are further classified as follows:

1. Spaces above the level of the hyoid bone (peritonsillar, submandibular, parapharyngeal, masticator, buccal, and parotid spaces).

2. Spaces that involve the entire length of the neck (retropharyngeal, prevertebral, and carotid spaces).

3. Spaces located below the level of hyoid bone (anterior visceral or pre - tracheal space).

Infection may present either as abscess, cellulitis, or necrotizing fasciitis. The mainstay of the management are antibiotics and surgical drainage.
Corresponding Author:

Dr. Priya Kanagamuthu, Department of Otorhinolaryngology, Chettinad Hospital and Research Institute, Kelambakkam, Tamil Nadu, India.

E-mail: catchpriya.29@gmail.com

DOI: $10.14260 / j e m d s / 2021 / 671$

How to Cite This Article:

Kanagamuthu P, Ramesh GK, Natarajan AV, et al. Deep neck space abscess - a case report. J Evolution Med Dent Sci 2021;10(37):3310-3313, $10.14260 /$ jemds $/ 2021 / 671$

Submission 03-06-2021,

Peer Review 24-08-2021,

Acceptance 30-08-2021,

Published 13-09-2021.

Copyright (c) 2021 Priya Kanagamuthu et al. This is an open access article distributed under Creative Commons Attribution License [Attribution 4.0 International (CC BY 4.0)] 


\section{PRESENTATION OF CASE}

A 66-year-old male presented with complaints of swelling over right side of neck for 5 days (FIG 1). Patient had history of dysphagia which was more for solids than liquids and pain over swelling was present which was dull aching in nature. Patient also had history of low-grade intermittent type of fever with headache before 5 days. History of hoarseness of voice, trismus, postnasal drip, cough with expectoration and sore throat were also noted. Patient had history of betel nut chewing for 15 years. Patient is a known case of diabetes for 5 years and systemic hypertension for 10 years.

On examination of the neck, diffuse swelling of size $5 \times 6$ $\mathrm{cm}$ was noted extending from right submandibular region 2 $\mathrm{cm}$ below hyoid bone to $2 \mathrm{~cm}$ above right clavicle, crossing midline and extending to left side of neck. Swelling was firm to hard in consistency. Warmth, tenderness and erythema were present. Skin over the swelling was pinchable.

Contrast enhanced computed tomography (CECT) neck revealed multiloculated collection with air pockets and surrounding inflammation involving right side of neck (FIG 2, 3).

Superolaterally, the collection involved parotid gland and parapharyngeal space adjacent to pterygoid muscle. Inferiorly it involved anterior belly of digastric on right side. Medially it involved lateral oropharyngeal wall. Posteriorly limited by internal jugular vein and carotid arteries. Extensive oedema was seen in nasopharynx, oropharynx and soft tissues of neck involving skin, subcutaneous tissue, right parotid and submandibular glands. Severe narrowing of oropharynx and nasopharyngeal airway was noted. Multiple enlarged nodes were seen on both sides of neck. Extensive vascular calcification was seen in intracranial carotid artery, vertebral arteries on both sides and basilar artery. Mild narrowing was seen in cavernous part of right internal carotid artery.

CT chest revealed patchy ground glass opacities with consolidatory changes in right lung and mosaic attenuation in bilateral lower lobes - CORADS 4.

After necessary investigations, patient was taken up for incision and drainage under general anaesthesia. Oblique neck incision was given parallel to the ramus of the mandible $2 \mathrm{~cm}$ below ramus. Incision was deepened and frank pus was evacuated (FIG 4) using blunt dissection. Masseteric space was opened to evacuate pus and superiorly blunt dissection was done up to parotid space. Inferiorly, subcutaneous plane blunt dissection was done. All identifiable locules were opened. Soft tissue was inflamed, and structure/plane of identification was difficult. Approximately $10 \mathrm{ml}$ of pus was collected and sent for culture sensitivity. Incision was given on the peritonsillar area on the right-side using Quinsy forceps and pus was drained. Thorough wash was given in the neck wound using saline, betadine and hydrogen peroxide. Wound cavity was packed with betadine-soaked gauze. Wound was left open (FIG 5) and dressing was done. Haemostasis was maintained throughout the procedure. Culture and sensitivity report revealed scanty growth of coagulase negative staphylococci.

Appropriate history, clinical examination and investigations led to the correct diagnosis and treatment plan. Patient was still on follow up and wound healing was good (FIG 6).
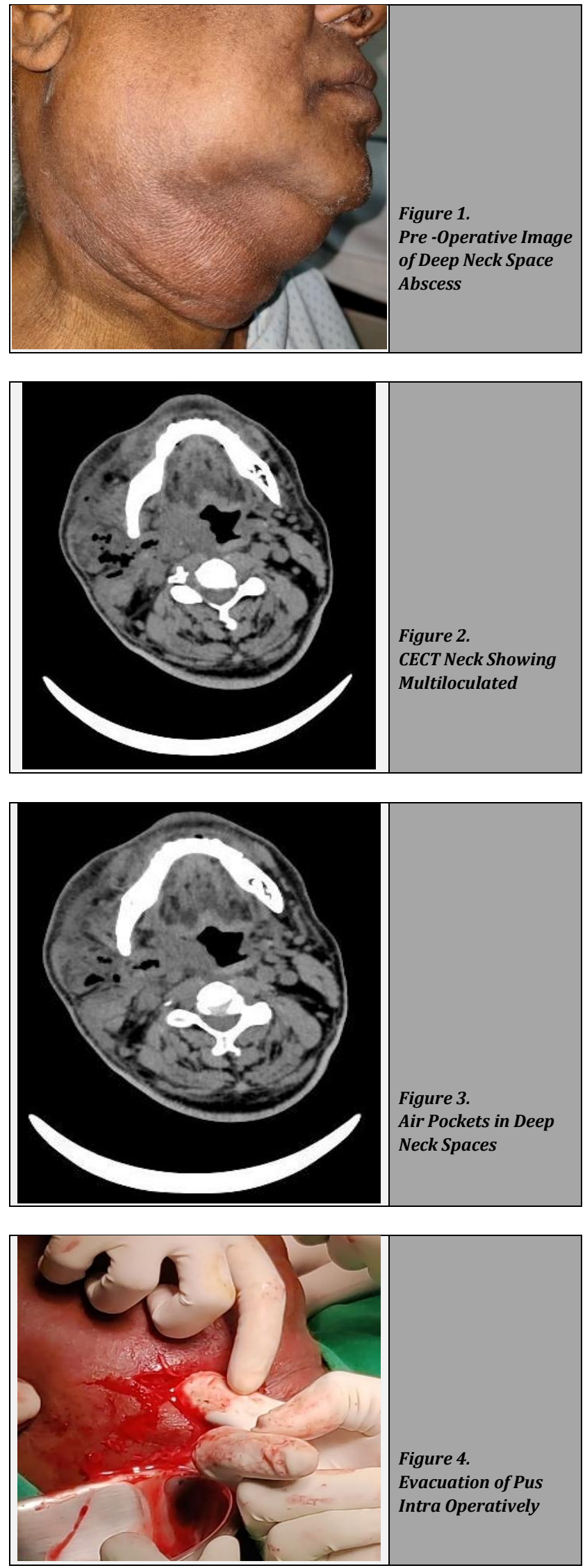

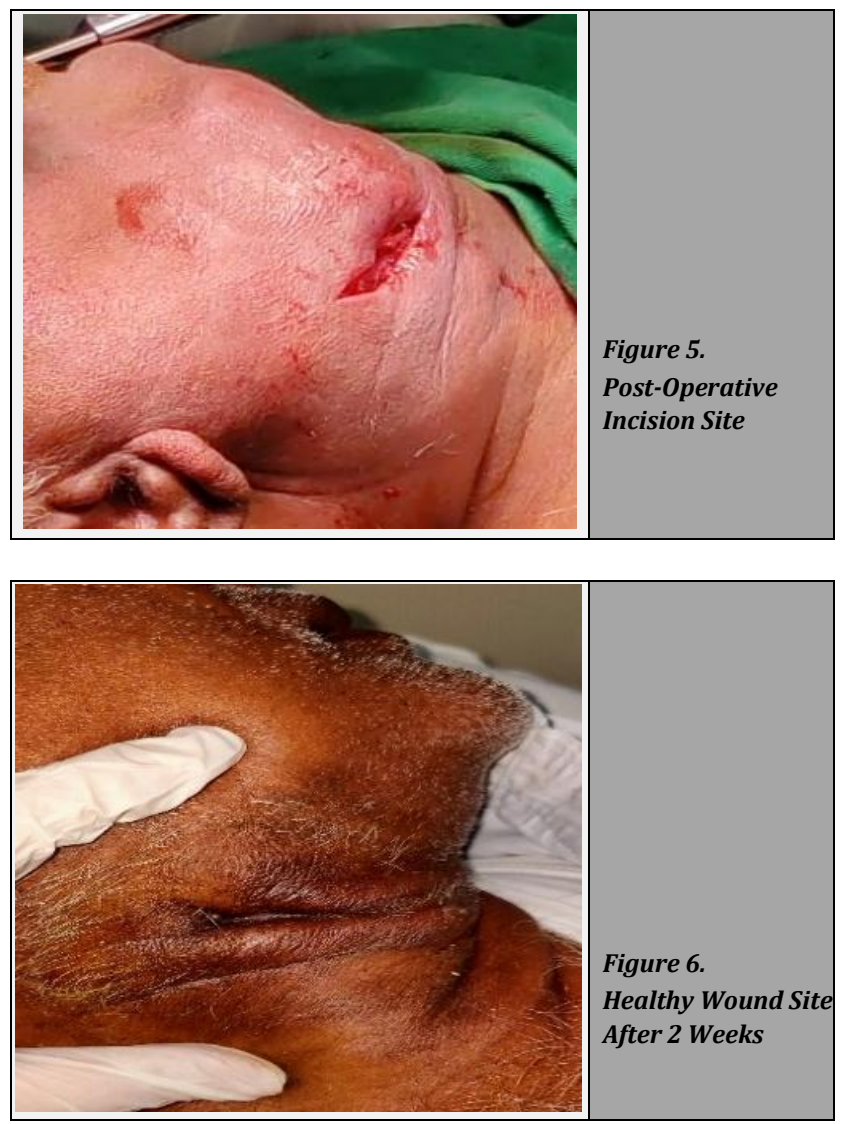

\section{DISCUSSION}

Poor oral hygiene due to tobacco smoking and betel nut chewing make the individual susceptible to various systemic diseases. Subgingival biofilms will be formed and serve as reservoirs for gram - negative organisms.

Odontogenic infections are the most common cause of infections in deep neck space, followed by tonsillopharyngitis. The most common site of deep neck space infection is the submandibular space followed by the peritonsillar space. Deep neck space abscesses involved in our study are parotid abscess and parapharyngeal abscess.

Usually, organisms isolated are normal flora from oropharynx. In this case, scanty growth of coagulase negative staphylococci with plenty of pus cells and occasional grampositive bacilli were seen. ${ }^{2}$

High-risk category such as geriatric population, immunocompromised patients, patients with diabetes mellitus and other systemic diseases should be given special attention. Because infections in deep neck spaces can also cause life threatening complications.

Radiological imaging confirms the clinical diagnosis, defines the extent of the disease and complications and it can distinguish between drainable abscesses and cellulitis and assess progress of the infection.

Abscess can be differentiated from cellulitis by the aid of ultrasonography which is considered to be gold standard investigation.

Ultrasonography also plays a role in the diagnosis of lymphadenitis and internal jugular vein thrombophlebitis in the infrahyoid region. ${ }^{3}$ Ultrasonography also plays a vital role in the diagnosis of abscess formation. ${ }^{4}$

Contrast enhanced CT combines fast image acquisition and anatomy without field view limitations. It also helps to differentiate fluid collection from cellulitis and detect vascular complications. CT scan also helps to decide the need for surgical intervention. Magnetic resonance imaging (MRI) plays a major role in assessing the epidural space involvement and complements CT in the evaluation of the skull base infections.

Treatment of deep neck space abscess is early surgical drainage of purulent abscess through an external incision and use of appropriate antibiotics. Empirical antibiotics should be given to treat both gram-positive and gram-negative infections, along with which intravenous fluids, analgesics, antipyretics and mouthwashes are given. Proper antibiotic coverage and timely surgical intervention will reduce complications and spread of infection.

Infections in the peritonsillar, submandibular, masticatory and parotid spaces can further spread to the parapharyngeal and retropharyngeal space. This spread can lead to several clinical presentations and deadly complications, depending on the space that is infected. ${ }^{6}$

The complications of deep neck space infections include septic shock, mediastinitis, pleural and pericardial effusion, thrombophlebitis of internal jugular vein, pulmonary embolism, cavernous sinus thrombosis, pseudo aneurysm or rupture of carotid artery, aortopulmonary fistula, epidural abscess, acute respiratory distress syndrome and disseminated intravascular coagulation. ${ }^{7}$

In patients with trismus and upper airway obstruction, airway management is crucial. Endotracheal intubation may be difficult in these patients due to limited access to the mouth. Hence whenever there is respiratory difficulty, tracheostomy should be preferred.

\section{CONCLUSIONS}

Deep neck space infections in developing countries are commonly seen among those individuals with lack of adequate nutrition, individuals with habit of tobacco and betel nut chewing and poor oral hygiene.

Quick diagnosis and treatment of deep neck space abscess is necessary for a good prognosis. All patients should be treated with intravenous antibiotic therapy initially and based on the culture and sensitivity report, susceptible drug therapy can be given later.

Surgical intervention is indicated in cases of significant abscess which is usually identified by CT scan.

Prevention of deep neck space infections can be done by creating awareness on dental and oral hygiene and doing regular dental check-ups.

Other differential diagnosis like tuberculosis, cat scratch disease, malignant tumours may also be a cause for the deep neck space infections and must be ruled out by doing the necessary investigations.

A large number of neck space infections are of dental origin, so the diagnosis and treatment should be done along with dental surgeons. ${ }^{8}$ Early surgical drainage is necessary for treating deep neck abscesses. ${ }^{9}$ 
Financial or other competing interests: None.

Disclosure forms provided by the authors are available with the full text of this article at jemds.com.

\section{REFERENCES}

[1] Priyamvada S, Motwani G. A study on deep neck space infections. Indian Journal of Otolaryngology and Head \& Neck Surgery 2019;71(Suppl 1):912-7.

[2] Rega AJ, Aziz SR, Ziccardi VB. Microbiology and antibiotic sensitivities of head and neck space infections of odontogenic origin. Journal of Oral and Maxillofacial Surgery 2006;64(9):1377-80.

[3] Maroldi R, Farina D, Ravanelli M, et al. Emergency imaging assessment of deep neck space infections. Seminars in Ultrasound, CT and MRI 2012;33(5):432-42.

[4] Kataria G, Saxena A, Bhagat S, et al. Deep neck space infections: a study of 76 cases. Iranian Journal of Otorhinolaryngology 2015;27(81):293-9.
[5] Bakir S, Tanriverdi MH, Gün R, et al. Deep neck space infections: a retrospective review of 173 cases. American Journal of Otolaryngology 2012;33(1):56-63.

[6] Pandey AK, Bansal C, Maithani T, et al. A perspective of clinical behaviour and management of Deep Neck Space Infections (DNSI): the clinical conundrum. Indian Journal of Otolaryngology and Head \& Neck Surgery 2019;71(Suppl 1):594-604.

[7] Lee JK, Kim HD, Lim SC. Predisposing factors of complicated deep neck infection: an analysis of 158 cases. Yonsei Medical Journal 2007;48(1):55-62.

[8] Stalfors J, Adielsson A, Ebenfelt A, et al. Deep neck space infections remain a surgical challenge. A study of 72 patients. Acta Otolaryngologica 2004;124(10):1191-6.

[9] Huang TT, Liu TC, Chen PR, et al. Deep neck infection: analysis of 185 cases. Head \& Neck: Journal for the Sciences and Specialties of the Head and Neck 2004;26(10):854-60. 